\title{
Rapid Progression of Tracheoesophageal Fistula Caused by Immunotherapy Administered after Tracheal Stent Placement
}

\author{
Shinjiro Mizuguchi Makoto Takahama Ryu Nakajima Hidetoshi Inoue \\ Ryuichi Ito Ryoji Yamamoto \\ Department of Thoracic Surgery, Osaka City General Hospital, Osaka, Japan
}

\begin{abstract}
What Is It about?
We report a rare case involving a patient with advanced lung adenocarcinoma invading the mediastinum who rapidly developed a tracheoesophageal fistula (TEF) after tracheal stent placement and nivolumab immunotherapy. Two weeks after the first dose of immunotherapy, mechanical debulking of the tumor with tracheal stenting was performed because of the rapid severe tracheal stenosis. Two weeks after the second dose, a huge TEF through metastatic lymph node was revealed. Our findings will be of great interest to the readers, especially those involved in the clinical treatment of patients with advanced lung cancer with transmural tracheal invasion treated by immunotherapy.
\end{abstract}

\section{Keywords}

Immunotherapy · Extensive invasion · Tracheoesophageal fistula · Lung cancer · Tracheal stent

\begin{abstract}
Development of a tracheoesophageal fistula (TEF) is a serious complication of treatment for esophageal or lung cancer, especially following radiation therapy. However, development of a TEF as a complication of chemotherapy or tracheal stenting after surgical debulking is quite uncommon. We herein report a rare case involving a patient with advanced adenocarcinoma invading the mediastinum who rapidly developed a TEF after placement of a tracheal stent and administration of nivolumab immunotherapy. A 55-year-old heavy ex-smoker was diagnosed with lung adenocarcinoma with mediastinal invasion. Nine months after first-line therapy (chemotherapy and radiation therapy), he underwent treatment with nivolumab $(3 \mathrm{mg} /$ $\mathrm{kg}$ ) as fourth-line therapy. Two weeks after the first dose, he underwent mechanical debulking of the tumor with tracheal stenting because of the rapid development of paraesophageal lymph node swelling and severe tracheal stenosis. He received a second dose of nivolumab 2 weeks later; however, imaging studies 12 days after this second dose revealed a huge fistula
\end{abstract}


between the upper trachea and esophagus through a metastatic lymph node. Neither an additional stent nor replacement of the stent was considered because of the fistula site expansion and suffocation risk. Despite further treatment, the patient died of his primary disease 2 months later. Our findings will be of great interest to the readers, especially those involved in the clinical treatment of patients with advanced lung cancer treated by immunotherapy. The knowledge of potentially devastating TEF formation in the presence of transmural tracheal metastasis/invasion will allow clinicians to provide the best possible care for their patients.

(C) 2019 The Author(s)

Published by S. Karger AG, Basel

\section{Established Facts}

- Pseudo-progression, tumor growth from treatment effect rather than true disease progression, has been described in immunotherapy.

- The potential of immunotherapy to mediate dramatic and rapid antitumor effects is well known in several malignant diseases.

\section{Novel Insights}

- To point out a potential safety concern: such anti-tumor effect occurring in patients with transmural metastasis/invasion of the trachea could have grave consequences.

\section{Introduction}

Nivolumab, a monoclonal antibody to the immune-checkpoint protein programmed cell death protein 1 , has been approved for the treatment of metastatic non-small cell lung cancer since having conferred a substantial survival benefit in phase III trials [1, 2]. Despite the possibility of an overly vigorous anti-solid tumor response, some reports have stated that only a single dose of nivolumab drastically eradicated the tumor $[3,4]$. An acquired tracheoesophageal fistula (TEF) is a life-threatening complication of esophageal or lung cancer. TEF is commonly reported as a complication of radiation therapy and rarely chemotherapy [5]. We herein describe rapid progression of a TEF in a patient with advanced adenocarcinoma invading the mediastinum treated with nivolumab before and after tracheal stent placement.

\section{Case Report}

A 55-year-old man presented for evaluation of dyspnea. He was a heavy ex-smoker (Brinkman index of 1,000). An initial imaging study revealed a lung tumor involving the mediastinum, and a biopsy revealed lung adenocarcinoma with an epidermal growth factor receptor wild-type and anaplastic lymphoma kinase-negative status (c-T4N0M0 stage 3A). The patient received concurrent chemotherapy (cisplatin and titanium silicate-1) and radiation therapy (60 Gy) to the mediastinum as first-line therapy. He was treated with several lines of cytotoxic agents, including carboplatin, nab-paclitaxel and pemetrexed, as well as additional radiation therapy for right supraclavicular and upper mediastinal lymph node metastasis. 
Fig. 1. First response of upper thoracic paraesophageal lymph node metastasis to nivolumab. Computed tomography scans showed rapid tumor progression before treatment (a) and 2 weeks after the first treatment (b), raising the possibility of "pseudo-progression."

Fig. 2. Bronchoscopy images before and after tracheal stenting and after one dose of nivolumab. a The bronchoscopy images showed tracheal stenosis and the tumor invading membranous trachea. b Electrocautery and mechanical debulking of the tumor were performed.c A silicone stent was placed after mechanical debulking of the tumor. After the second dose of nivolumab, the stent was migrated to necrotic tissue (d) and a huge fistula between the upper trachea and upper part of esophagus was revealed (e). $\mathbf{f}$ The fistula opening was clearly visible and had expanded 3 weeks after the second dose of nivolumab.

Fig. 3. Tracheoesophageal fistula through a necrotic paraesophageal lymph node after the second dose of nivolumab. a, d Computed tomography images showed the tracheoesophageal fistula at the proximal side of the placed stent. b, c A 3-cm segment of the membranous trachea had disappeared, and the stent was migrated.
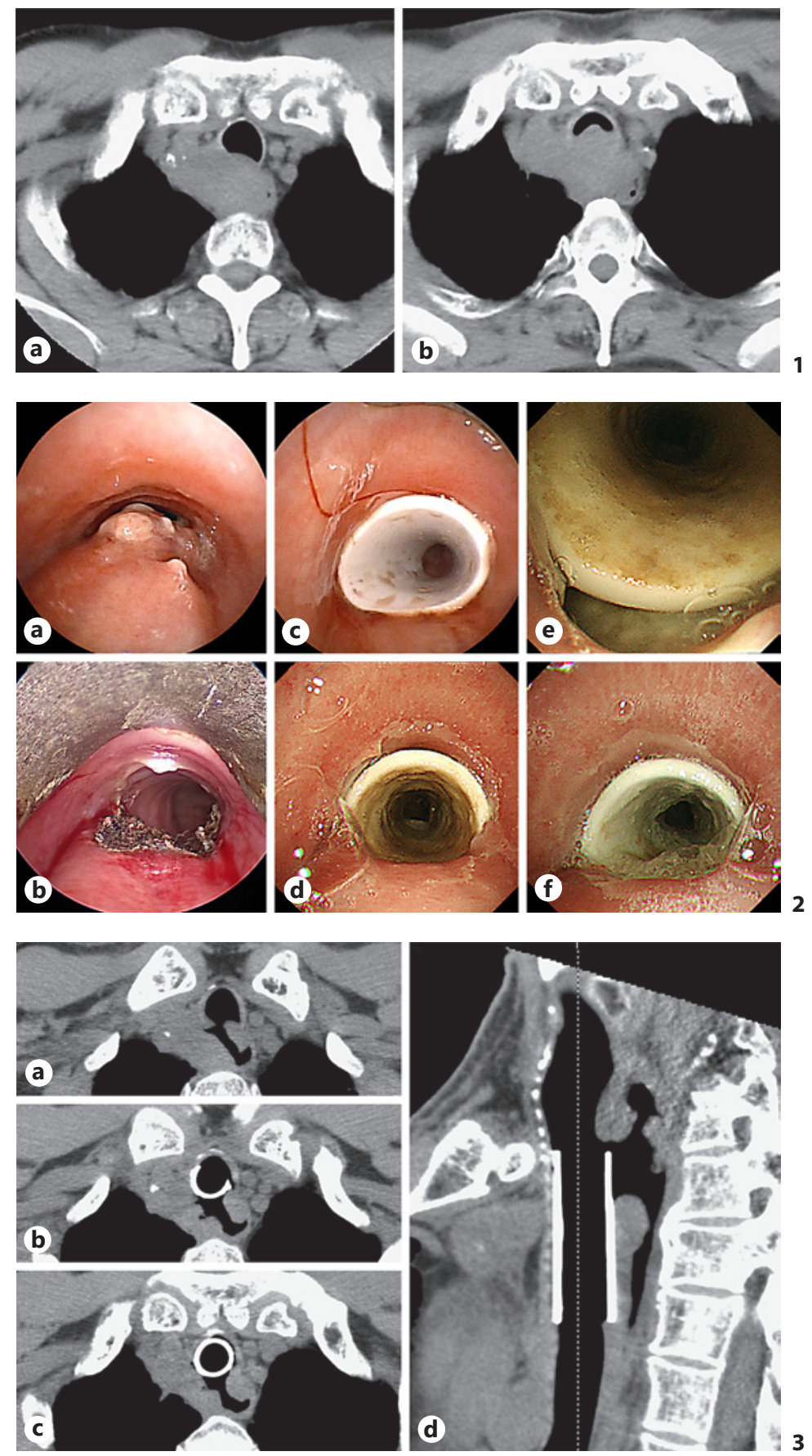

Nine months after the first-line treatment, he received nivolumab (3 mg/kg) as fourthline therapy. Two weeks after his first dose of nivolumab, he was referred for dysphagia and severe dyspnea. Imaging examination showed rapid progression of paraesophageal lymph node swelling and severe stenosis of the main trachea (Fig. 1). Because his performance status and Hugh-Jones classification had worsened to 3 and IV, respectively, mechanical debulking of the tumor arising from the membranous trachea was performed, and a silicone stent of 18-mm diameter and 50-mm length (TRACHEOBRONXANE ${ }^{\text {TM }}$ DUMON $^{\circledR}$ TD; Novatech $\mathrm{SA}$, La Ciotat, France) was placed using rigid bronchoscopy (Fig. 2a-c). Two weeks after 
stenting, the patient received a second dose of nivolumab. Recurrent aspiration occurred 10 days after this dose, and he was admitted 2 days later. Chest computed tomography and bronchoscopy revealed a huge fistula between the upper trachea and esophagus through a metastatic lymph node, which was $20-30 \mathrm{~mm}$ in size (Fig. 2d, e; Fig. 3). The fistula, both the proximal and distal side of the silicone stent (Fig. 2f), had expanded and was clearly visible after 1 week. Neither an additional stent nor replacement of the stent was considered because of the fistula site expansion and suffocation risk. The patient underwent gastrostomy and antibiotic therapy. However, he died of the primary disease 2 months later.

\section{Discussion}

The reported incidence of malignant TEF in patients with lung cancer ranges from 0.16 to $0.30 \%[6,7]$. In one study, the median survival time from diagnosis was only 6 weeks in the absence of appropriate treatment [6]. TEF is commonly reported as a complication of radiation therapy and rarely chemotherapy [5], and various anti-angiogenic agents have been found to increase the risk of fistula formation [8], especially bevacizumab; such agents can cause delayed healing secondary to impaired angiogenesis according to a previous review [9]. TEF formation is rare not only following immunotherapy but also tracheal stenting after tumor debulking of an invasive tracheal tumor.

The patient in the present report experienced rapid eradiation of a large tumor after two treatments with immunotherapy. Although no histological assessment was performed, the progression of the tumor after the first course of immunotherapy that caused dyspnea and tracheal stenosis might have been "pseudo-progression"; i.e., initial tumor growth followed by tumor regression. A previous review paper showed that approximately $4 \%$ of patients with solid tumors who received continued anti-programmed death-ligand 1 treatment after RECIST tumor progression obtained an anti-tumor response [10]. Although our patient underwent mechanical debulking of his rapidly progressing tumor followed by tracheal silicone stenting, it might have been more suitable to use a covered self-expandable metal stent without debulking in view of the risk of TEF following rapid tumor necrosis. The tracheal walls that may have been invaded by the tumor had rapidly necrotized, leading to their collapse and subsequent fistula formation. This rare case highlights that such an anti-tumor effect occurring in patients with transmural metastasis/invasion of the trachea could have grave consequences.

\section{Acknowledgment}

We thank Angela Morben, DVM, ELS, from Edanz Group (www.edanzediting.com/ac), for editing a draft of the manuscript.

\section{Statement of Ethics}

Written informed consent was obtained from the patient and his family for publication of this case report and any accompanying images.

\section{Disclosure Statement}

The authors declare no conflicts of interest. 


\section{References}

1 Brahmer J, Reckamp KL, Baas P, Crinò L, Eberhardt WE, Poddubskaya E, et al. Nivolumab versus Docetaxel in Advanced Squamous-Cell Non-Small-Cell Lung Cancer. N Engl J Med. 2015 Jul;373(2):123-35.

2 Borghaei H, Paz-Ares L, Horn L, Spigel DR, Steins M, Ready NE, et al. Nivolumab versus Docetaxel in Advanced Nonsquamous Non-Small-Cell Lung Cancer. N Engl J Med. 2015 Oct;373(17):1627-39.

3 Chapman PB, D'Angelo SP, Wolchok JD. Rapid eradication of a bulky melanoma mass with one dose of immunotherapy. N Engl J Med. 2015 May;372(21):2073-4.

4 Davar D, Socinski MA, Dacic S, Burns TF. Near complete response after single dose of nivolumab in patient with advanced heavily pre-treated KRAS mutant pulmonary adenocarcinoma. Exp Hematol Oncol. 2015 Dec;4(1): 34.

5 Perry RR, Rosenberg RK, Pass HI. Tracheoesophageal fistula in the patient with lymphoma: case report and review of the literature. Surgery. 1989 Jun;105(6):770-7.

6 Reed MF, Mathisen DJ. Tracheoesophageal fistula. Chest Surg Clin N Am. 2003 May;13(2):271-89.

7 Martini N, Goodner JT, D’Angio GJ, Beattie EJ Jr. Tracheoesophageal fistula due to cancer. J Thorac Cardiovasc Surg. 1970 Mar;59(3):319-24.

8 Goodgame B, Veeramachaneni N, Patterson A, Govindan R. Tracheo-esophageal fistula with bevacizumab after mediastinal radiation. J Thorac Oncol. 2008 Sep;3(9):1080-1.

9 Nishie K, Yasuo M, Kitaguchi Y, Kobayashi N, Tateishi K, Ushiki A, et al. Bevacizumab-induced tracheoesophageal fistula in a patient suffering from lung cancer with bulky subcarinal lymph node: a case report. Nagoya J Med Sci. 2018 Feb;80(1):129-34.

10 Chiou VL, Burotto M. Pseudoprogression and Immune-Related Response in Solid Tumors. J Clin Oncol. 2015 Nov;33(31):3541-3. 